\title{
Discontinuous Interface Depinning from a Rough Wall
}

\author{
G. Giugliarelli \\ Dipartimento di Fisica e INFN (Gruppo Collegato di Udine, Sezione di Trieste), Universitá di \\ Udine, 33100 Udine, Italy \\ A. L. Stella \\ INFM - Dipartimento di Fisica e Sezione INFN, Universitá di Padova, 35131 Padova, Italy
}

\begin{abstract}
Depinning of an interface from a random self-affine substrate with roughness exponent $\zeta_{S}$ is studied in systems with short-range interactions. In $2 D$ transfer matrix results show that for $\zeta_{S}<1 / 2$ depinning falls in the universality class of the flat case. When $\zeta_{S}$ exceeds the roughness $\left(\zeta_{0}=1 / 2\right)$ of the interface in the bulk, geometrical disorder becomes relevant and, moreover, depinning becomes discontinuous. The same unexpected scenario, and a precise location of the associated tricritical point, are obtained for a simplified hierarchical model. It is inferred that, in $3 D$, with $\zeta_{0}=0$, depinning turns first-order already for $\zeta_{S}>0$. Thus critical wetting may be impossible to observe on rough substrates.
\end{abstract}

PACS numbers: 64.60.Ak, 68.45.Gd, 36.20.Ey, 75.60.Ch

Typeset using REVTEX 
Wetting and depinning phenomena occur when the interface between two coexisting phases unbinds from an attractive substrate [1]. In $2 D$, where an Ising interface can be well represented by a directed path [2], critical wetting occurs as a rule, and first-order wetting is predicted only for special setups [1]. In 3D, on the other hand, numerical simulations have shown critical, first-order and tricritical wetting for the Ising model. The same phenomena in random media have attracted a lot of attention recently. Many works [3] 5] considered the effects of quenched disorder due to bulk impurities on interface depinning. Other studies addressed disorder restricted to a geometrically smooth surface (chemical surface disorder) [6]. Both bulk and chemical surface disorder may modify the universality class of the unbinding transition, but generally not its continuous, second-order character in 2D. [1]

In the present Letter we address the role of geometrical disorder due to wall roughness in determining the nature of the wetting transition. Disordered geometry is most amenable to theoretical treatment when characterized by simple scaling laws, like in the case of self-affine or fractal substrates. So far, the effects of such roughness were seldom discussed, mostly in connection with complete wetting [7 9]. In spite of this, real substrates with self-affine geometry are met in many situations and have been the object of recent experiments [8]. For these substrates the average transverse width of the wall scales with the longitudinal length $X$ as $X^{\zeta_{S}}\left(0<\zeta_{S}<1\right)$.

We show that in the $2 D$ Ising model the nature of the depinning transition changes drastically upon increasing the self-affine roughness exponent $\zeta_{S}$. For $\zeta_{S} \lesssim 1 / 2$ depinning remains continuous as in the flat case, and disorder is irrelevant. For $\zeta_{S} \gtrsim 1 / 2$ an unusual discontinuous depinning occurs. Since the intrinsic roughness of a $2 D$ interface is $\zeta_{0}=1 / 2$, it is natural to expect $\zeta_{S}=1 / 2$ as precise threshold. The emerging scenario is that of geometrical disorder determining a tricritical phenomenon for depinning. We are able to locate the tricritical point by accurate renormalization group ( $R G$ ) calculations on a hierarchical model, which further suggest $\zeta_{S}=1 / 2$ as the tricritical threshold in $2 D$. Extrapolation of our results to the experimentally most relevant case of $3 D$, where $\zeta_{0}=0$ in ordered bulk, suggests that a minute disorder in substrate geometry could suppress critical wetting in favor of a first-order transition.

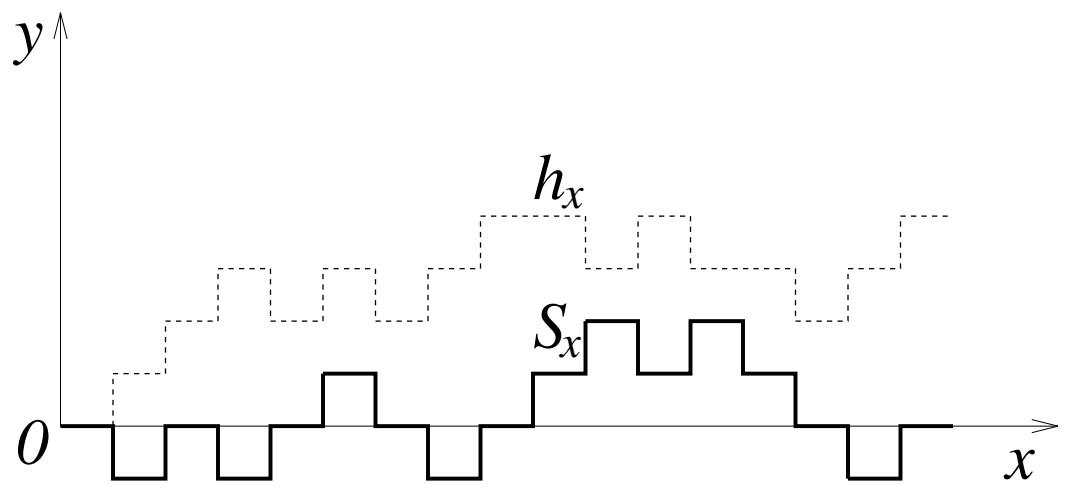

FIG.1. Example of rough substrate boundary (continuous) and interface (dotted) configuration.

Let $x$ and $y$ be integer coordinates of points on a square lattice. A "wall" is given by 
a self-avoiding path directed according to the positive $x$ axis (Fig. 1). To each wall step parallel to the $x$ axis, a height $S_{x}$, equal to the ordinate of its left end, $x$, is associated. For simplicity we consider only wall configurations obeying the restriction: $s_{x}=S_{x}-S_{x+1}= \pm 1$. In order to generate wall geometries with a preassigned roughness exponent $\zeta_{S}$, we used a randomized version of an algorithm due to Mandelbrot [10]. Given a wall profile, the interface can assume configurations obeying $h_{x}-h_{x+1}=0, \pm 1$ and $h_{x} \geq S_{x}$, if $h_{x}$ is the height of the horizontal step at $x$ (Fig. 1). The interface is like a (partially) directed walk or polymer, and its Hamiltonian is

$$
\mathcal{H}=\sum_{x}\left[\varepsilon\left(1+\left|z_{x}-z_{x+1}+s_{x}\right|\right)-u \delta_{z_{x}, 0}\right]
$$

where $z_{x} \equiv h_{x}-S_{x}$, and $\varepsilon, u>0$. According to (1), at temperature $T$, fugacities $\omega \equiv e^{-\varepsilon / T}$ and $k \equiv e^{u / T}$ are associated to each (horizontal or vertical) step of the walk, and to each horizontal step on the wall, respectively. With a wall profile covering a distance $X$ the partition function is

$$
\mathcal{Z}_{X}=\sum_{\text {all walks }} \omega^{L} k^{l}
$$

where the sum is restricted, e.g., to walks from the origin $(0,0)$ to any point $(X, y)$, with $y \geq$ $S_{X} . L$ and $l$ indicate total length and number of horizontal steps on the wall, respectively. $\mathcal{Z}_{X}$ is a functional of the wall profile. In order to calculate it we use transfer matrices:

$$
\left(\mathbf{T}_{s_{x}}\right)_{m, n}=\omega\left[\delta_{n, m-s_{x}}+\left(\delta_{n, m-s_{x}-1}+\delta_{n, m-s_{x}+1}\right) \omega\right] k^{\delta_{n, 0}}
$$

where $m$ and $n$ range on the allowed $z_{x}$ and $z_{x+1}$, respectively. The partition function thus becomes

$$
\mathcal{Z}_{X}=\sum_{l, p}\left(\prod_{x=0}^{X-1} \mathbf{T}_{s_{x}}\right)_{l, p} \phi_{0}(p)
$$

where, with the left end of the interface grafted at the origin, $\phi_{0}(p)=\delta_{p, 0}$. A wall profile corresponds to a sequence of factors $\mathbf{T}_{1}, \mathbf{T}_{-1}$ in the product of eq.(4). $\mathcal{Z}_{X}$ is thus given asymptotically in terms of the largest Lyapunov eigenvalue [1]

$$
\lambda_{\max }=\lim _{X \rightarrow \infty}\left[\frac{\left\|\left(\prod_{x=0}^{X-1} \mathbf{T}_{s_{x}}\right) \vec{\phi}_{0}\right\|}{\left\|\vec{\phi}_{0}\right\|}\right]^{\frac{1}{X}}=\lim _{X \rightarrow \infty}\left[\frac{\left\|\vec{\phi}_{X}\right\|}{\left\|\vec{\phi}_{0}\right\|}\right]^{\frac{1}{X}}
$$

We verified that different long $\mathbf{T}$ sequences, i.e. wall profiles, lead to the same eigenvalue within good accuracy. Thus, the quenched free energy is $\ln \lambda_{\max }\left(\omega, k, \zeta_{S}\right)=$ $\lim _{X \rightarrow \infty} \overline{\ln \mathcal{Z}_{X}} / X$, where the bar indicates quenched averaging over wall profiles. In the random context depinning is most efficiently detected by studying the behavior of quantities which can be directly related to the components of $\vec{\phi}_{X}$. Examples are the average probability, $\overline{P_{0}}\left(\omega, k, \zeta_{S}\right)=\lim _{X \rightarrow \infty} \overline{\frac{1}{X} \sum_{x=0}^{X-1} \phi_{x}^{2}(0) /\left\|\vec{\phi}_{x}\right\|^{2}}$, that the horizontal step lies on the wall, and the average distance of the interface from the substrate, $\overline{\langle z\rangle}=\lim _{X \rightarrow \infty} \overline{\frac{1}{X} \sum_{x=0}^{X-1} \sum_{z} z \phi_{x}^{2}(z) /\left\|\vec{\phi}_{x}\right\|^{2}}$ 11. For our determinations we used up to 50 independent profiles with $x \leq 2^{20}$ for which the components of $\vec{\phi}_{x}$ were computed up to a distance from the wall $z_{\max } \simeq 2 \cdot 10^{4}$. 
TABLE I. Values of $\psi$ and $k_{c}$ at $\omega=1 / 2$ from fits of $\overline{\langle z\rangle}$.

\begin{tabular}{ccc}
\hline$\zeta_{S}$ & $\psi$ & $k_{c}$ \\
\hline 0 & 1 & $4 / 3^{a}$ \\
$1 / 3$ & $1.01 \pm 0.01$ & $1.772 \pm 0.001$ \\
$2 / 5$ & $1.01 \pm 0.02$ & $1.828 \pm 0.001$ \\
$1 / 2$ & $1.03 \pm 0.04$ & $1.908 \pm 0.003$ \\
\hline
\end{tabular}

a Exact results for a flat substrate [1].

Rather than considering variations of $\overline{P_{0}}$ or $\overline{\langle z\rangle}$ along curves parametrized by temperature, we choose to follow $\omega=$ const. lines. In the case $\zeta_{S} \lesssim 1 / 2$, e.g., upon approaching $k=k_{c}$ from above with $0<\omega<1, \overline{\langle z\rangle}$ is well fitted by $\overline{\langle z\rangle}=A\left(k-k_{c}\right)^{-\psi}+B$, with $\psi$ always compatible with the exactly known flat wall value of 1 [ [] Some $\psi$ and $k_{c}$ determinations are reported in Table I for $\omega=1 / 2$. For $\zeta_{S} \lesssim 1 / 2$ disorder in the wall geometry does not appear to lead to a new universality class for depinning. By treating a different model with continuum many-body techniques, Li and Kardar [7] found second-order depinning with $\psi=1$ for all $\zeta_{S}<1$. At variance with this conclusion we find here that the situation drastically changes for $\zeta_{S} \gtrsim 1 / 2$. In this range $\overline{\langle z\rangle}$ has a much steeper, abrupt rise at $k_{c}$, so that the previous fit becomes clearly inappropriate.

Further strong evidence of a change of the nature of the transition at $\zeta_{S} \simeq 1 / 2$ comes from the behavior of $\overline{P_{0}}$ (Fig. 2). While for $\zeta_{S} \lesssim 1 / 2, \overline{P_{0}} \sim\left(k-k_{c}\right)^{\rho}$, for $k \rightarrow k_{c}^{+}$, with $\rho \simeq 1$, as with flat substrate [1], for $\zeta_{S} \gtrsim 1 / 2$ a discontinuity in $\overline{P_{0}}$ shows up (Fig. 2), becoming sharper and sharper as finite-size effects are reduced. The amplitude of the discontinuous jump in $\overline{P_{0}}$ also increases with $\zeta_{S}$.

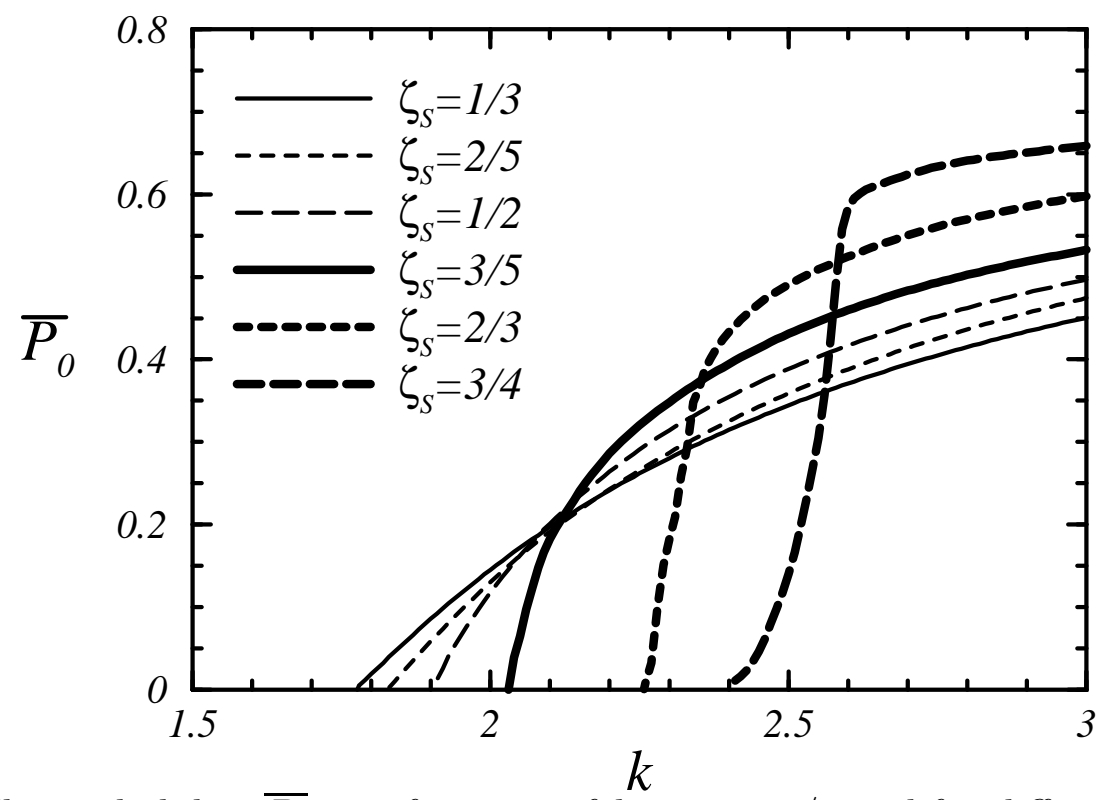

FIG. 2. The probability $\overline{P_{0}}$ as a function of $k$ at $\omega=1 / 2$ and for different $\zeta_{S}$ values. 
Thus, for $\zeta_{S} \gtrsim 1 / 2$ substrate roughness is relevant and, moreover, drives the depinning transition first-order. $\zeta_{S}=1 / 2$ is the natural candidate as border value between continuous and discontinuous regimes. Indeed, for $\zeta_{S}>1 / 2$ the wall roughness exceeds the roughness $\zeta_{0}=1 / 2$ [5] of the interface.

In $2 D$, first-order depinning is quite unexpected in the context of interfacial phenomena. Only two special ways of obtaining it have been conceived so far, by introducing either an attractive defect line in the bulk [12], or longitudinally fully correlated disorder [13]. Here first order is caused by sufficiently strong geometrical surface disorder, which also reveals opposite in its effects to its chemical counterpart. Indeed, while higher roughness induces first-order, in the defect line case chemical surface disorder drives the transition back continuous [14]. This latter effect is certainly what one would expect at first sight on the basis of experience with phase transitions [15]. Like in the special examples of refs. 12,13, our first order depinning needs not be accompanied by off-coexistence prewetting phenomena.

Extrapolation to $3 D$ of our findings is natural and has remarkable and unexpected consequences. Systems with a dominance of short-range interactions are, e.g., metallic substrateadsorbates or, even more, type-I superconductors [16]. Since typically interfaces in pure systems are only logarithmically rough in $3 D\left(\zeta_{0}=0\right)$, a minimum of substrate roughness should be sufficient to give first-order wetting. This offers a further possible explanation of the fact that critical wetting is so elusive from the experimental point of view. A most recent work predicts critical wetting for superconductor interfaces [16]. For such interfaces $\zeta_{0}$ is not known, unfortunately, but could be rather small, if not zero. This means that special care in using smooth substrates should be exerced, in order to observe the predicted phenomenon.

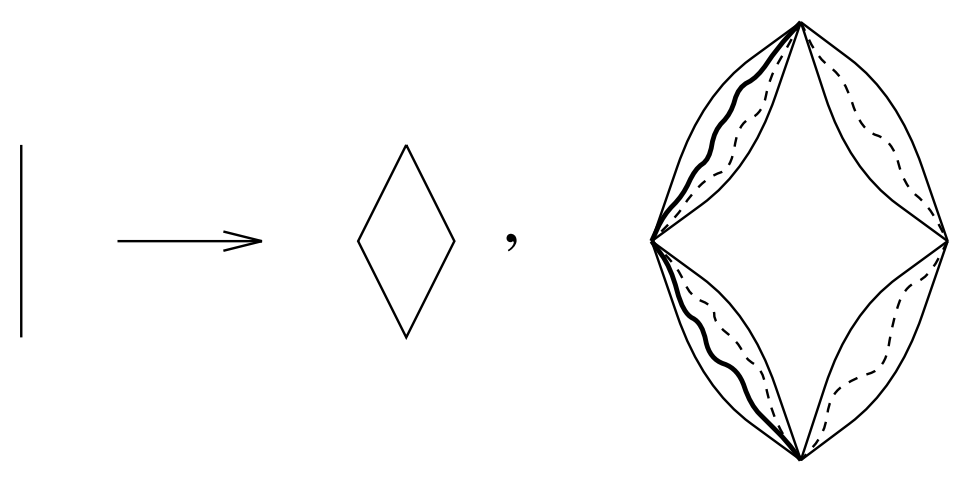

FIG. 3. Construction rule of the DHL (level $n=0$ to level $n=1$ ) and schematic picture of the lattice at level $n$, with the four $n-1$ level units. A wall configuration (heavy) crossing the left units, and two polymer configurations (dotted) are reported. With such wall configuration eq. (6) applies.

By reinterpreting $\omega$ and $k$ as monomer fugacity and Boltzmann factor for contacts, respectively, our model describes polymer adsorption [17]. Apart from a change in the ensemble $\left(\mathcal{Z}=\sum_{X} \mathcal{Z}_{X}\right)$, the transfer matrices are the same. Criticality ( $\mathcal{Z}$ dominated by infinite length polymer configurations) implies $\lambda_{\max }=1$. We find that $\lambda_{\max }\left(1 / 2, k, \zeta_{s}\right)=$ 
1 for all $k<k_{c}\left(1 / 2, \zeta_{S}\right)$, where $k_{c}$ was defined above. For $k>k_{c}$ criticality occurs at $\omega<1 / 2$, indicating that the polymer is adsorbed [17]. Indeed $\omega=1 / 2$ marks criticality for the polymer in the bulk. The dependence of $k_{c}$ on $\zeta_{S}$ shows that adsorption becomes more difficult with increasing roughness. One can also show that the fraction of monomers adsorbed on the wall should have the same singular behavior as $\overline{P_{0}}$, when moving at $\omega=1 / 2$. Thus, like interface depinning, polymer adsorption undergoes a change from second to first order upon increasing $\zeta_{S}$.

To gain more insight into this change, we stick to polymer language and consider a simplified model of adsorption defined on diamond hierarchical lattice (DHL) (Fig. 3). Selfavoiding paths on DHL have often been used to mimic directed polymers in $2 D[18]$. A wall joining the two ends is obtained as follows: at level $n=0$ of DHL construction the wall always coincides with the unique existing bond. For $n=n_{\max }>0$, the wall is determined by backward iteration. Starting from $n_{\max }$, at each level, $n$, we choose whether the wall passes through the left or right DHL units of level $n-1$, with probabilities $1-\Delta$ and $\Delta$, respectively, and so on. If, e.g., we put $\Delta=0$, the process is deterministic and we create a single wall coinciding with the left border of the lattice. For $\Delta=1 / 2$ we generate with equal probability all possible walls through the lattice. Given a wall, we consider a polymer, with partition $\mathcal{Z}_{n}$, joining the ends of the lattice and laying, e.g., to the right of the wall. As in eq. (2), $\omega$ and $k$ are step and wall contact fugacities, respectively. For $\Delta=0$ we deal with a polymer attracted $(k>1)$ by the left DHL border, which plays the role of a "flat", deterministic substrate. When $\Delta$ rises, the "roughness" of the now random wall increases. Transverse hills and valleys are felt more and more by the polymer. For a given wall, one can compute the polymer partition function iteratively, using $\mathcal{Z}_{b, n+1}=2 \mathcal{Z}_{b, n}^{2}$ and

$$
\mathcal{Z}_{n+1}=\mathcal{Z}_{n, 1} \mathcal{Z}_{n, 2}+\mathcal{Z}_{b, n}^{2}
$$

or

$$
\mathcal{Z}_{n+1}=\mathcal{Z}_{n, 1} \mathcal{Z}_{n, 2}
$$

Eq.(6) or (7) is chosen, according to whether, at level $n+1$, the $n$-th level diamonds, 1 and 2 , crossed by the wall, are the left or the right ones, respectively. $\mathcal{Z}_{b, n}$ is clearly the "bulk" partition function on DHL at $n$-th level, in absence of wall. Initial conditions are $\mathcal{Z}_{0}=k \omega$ and $\mathcal{Z}_{b, 0}=\omega$. Considering first $\Delta=0$, eq.(6) induces a two parameters RG mapping by putting $\mathcal{Z}_{b, 1}=\omega^{\prime}$ and $\mathcal{Z}_{1}=\omega^{\prime} k^{\prime}$. Clearly the transformation of $\mathcal{Z}_{b, n}$ implies that the bulk criticality condition is $\omega=1 / 2$ as in the Euclidean case. The value $k=1$ is marginally unstable for $k \gtrsim 1$ and separates adsorbed $(k>1)$ from desorbed $(k<1)$ regimes. Thus, for $\Delta=0$ a positive attraction is always sufficient to adsorb the polymer. Eq. (6) applies for all $n$ and the problem has a nontrivial fixed point with $\mathcal{Z}_{b}^{*}=1 / 2$ and $\mathcal{Z}^{*}=1 / 2 . \quad P_{0}(\omega=1 / 2, k, \Delta=0)$ can be extrapolated by iteration. Due to marginality, $P_{0}$ starts rising with zero slope, but continuously, for $k=1$ (Fig. 4). For $\Delta>0$ the $\mathcal{Z}_{n}$ 's become random variables and we must iterate their probability distribution, $\mathcal{P}_{n}$. This cannot be done exactly. However, starting from $\mathcal{P}_{0}=\delta(\mathcal{Z}-k \omega)$, we follow the distribution at level $n$ by iteratively sampling it. From a large number $\left(\simeq 10^{6}\right)$ of $\mathcal{Z}$ values distributed according to $\mathcal{P}_{n-1}$, we generate a sample distributed according to $\mathcal{P}_{n}$ by choosing many pairs 
of $\mathcal{Z}$ values and obtaining from each pair a new value of $\mathcal{Z}$ according to eq.(6) or (7), with probabilities $1-\Delta$ and $\Delta$, respectively. The resulting $\mathcal{Z}$ 's constitute a sample of $\mathcal{P}_{n}$. This procedure could be iterated for $n \leq 40$ with extremely high accuracy.

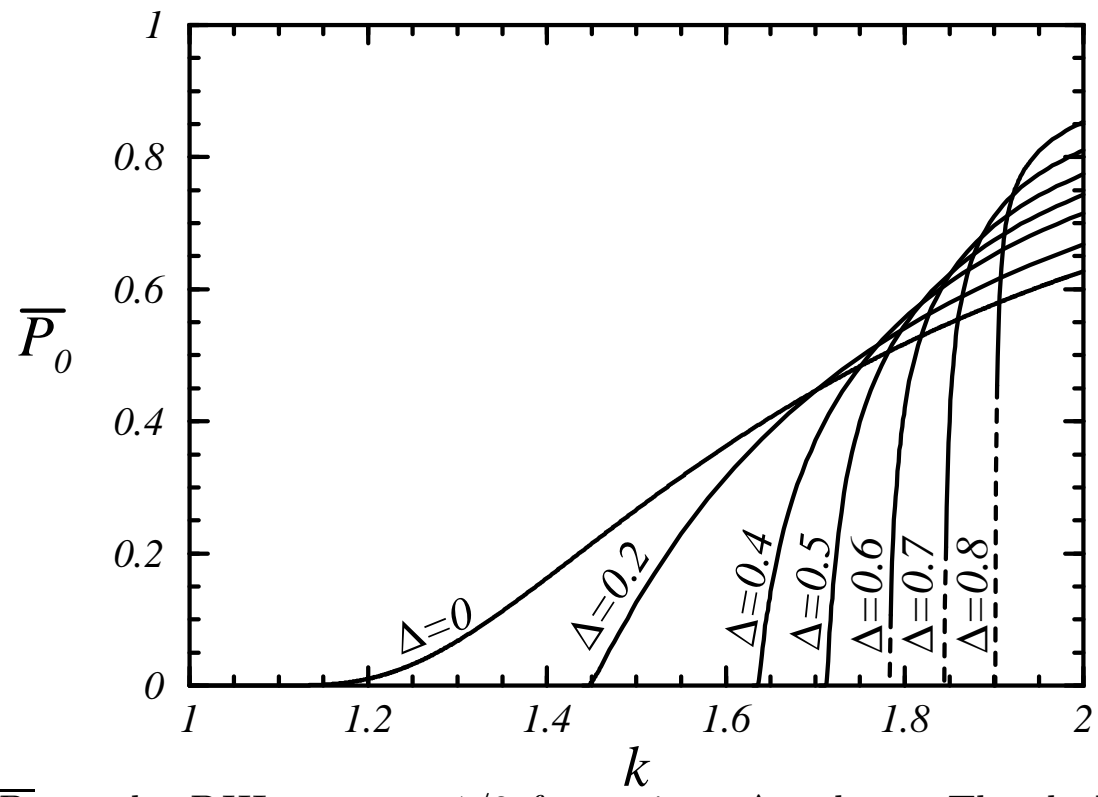

FIG. 4. $\overline{P_{0}}$ on the DHL at $\omega=1 / 2$ for various $\Delta$ values. The dashed lines mark the discontinuities for $\Delta>1 / 2$.

$\overline{P_{0}}$ at $\omega=1 / 2$ is plotted as a function of $k$ in Fig. 4 for different $\Delta$ 's. For $0<\Delta<1 / 2$, $\overline{P_{0}}$ rises continuously from zero as $c\left(k-k_{c}\right)$. Thus, $\rho=1$ for $0<\Delta<1 / 2$. The slope $c$ and $k_{c}$ are both increasing with $\Delta$. Apart from $c=0$ at $\Delta=0$, due to the accidental marginality, such behaviour reproduces what is observed in $2 D$ when $\zeta_{S}<1 / 2$. For $\Delta<1 / 2$ the polymer is more "rough" than the wall. Only when $\Delta=1 / 2$ the latter has the same freedom to develop through the DHL as a polymer has within the "bulk". On the other hand, $\Delta>1 / 2$ corresponds qualitatively to $\zeta_{S}>1 / 2$, because the polymer feels more and more the wall limiting its options when developing through the DHL. For $\Delta=1 / 2$ we have evidence that $c=\infty$, with a still continuous transition. This infinite slope indeed anticipates a sharp discontinuity in $\overline{P_{0}}$ for $\Delta>1 / 2$. So, the hierarchical model contains ingredients reproducing, at least qualitatively, the scenario emerging for the Euclidean model, and gives a suggestive indication of the way in which continuous transitions switch to first order at the expected threshold $\zeta_{S}=1 / 2$.

The dependence of $k_{c}$ on $\Delta$ mimics that on $\zeta_{S}$ in $2 D$, and further motivates the correspondence between $\Delta$ and $\zeta_{S}$ in the two cases. We stress that, since all paths have the same length, and there is no natural recipe for defining a transversal distance on DHL, the notion of roughness must always be mediated in some way: here we can link roughness to $\Delta$. Our hierarchical model provides a remarkable example of the tricritical transition we are dealing with in this paper, and allows an essentially exact determination of its location and properties. Moreover, the threshold we find at $\Delta=1 / 2$ is strongly suggestive of the precise location of the tricritical point in the Euclidean case.

Summarizing, in $2 D$, interface depinning or directed polymer adsorption on rough sub- 
strate with $\zeta_{S}<1 / 2$ are continuous and in the same universality as in the flat case. For $\zeta_{S}>1 / 2$ roughness is relevant and, moreover, the transitions acquire an unusual, discontinuous nature. This result, not anticipated so far [7], warns that some continuum approaches may not be able to catch the correct physics of depinning from rough substrates. We expect similar properties, and the same threshold $\zeta_{S}=1 / 2$, for directed polymer adsorption on a self-affine surface in $3 D$. Indeed, directed polymers have no upper critical dimension, and for them $\zeta_{0}=1 / 2$ in all $d$. Although the single polymer adsorption regime is not easily accessible experimentally, we believe that our results should be relevant for stretched polymers 19 .

In $3 D$ the interface of a pure system typically has $\zeta_{0}=0$ [5]. We thus conjecture that depinning occurs discontinuously as soon as $\zeta_{S}>0$, in cases when the interactions are predominantly short-range, like in metallic systems. Our results also bear on the observability of the recently predicted critical wetting in the case of type-I superconductors [16], which is perhaps the most strict physical example of short-range interface-substrate interactions.

Numerical calculations have been partly supported by CNR within the CRAY project of Statistical Mechanics. We thank Joseph Indekeu for valuable criticism and suggestions and Mehran Kardar for stimulating discussions. 


\section{REFERENCES}

[1] G. Forgacs, R. Lipowsky and Th.M. Nieuwenhuizen, in "Phase Transitions and Critical Phenomena", by C. Domb and J.L. Lebowitz, Vol. 14, Academic Press, 1991.

[2] S.T. Chui and J.D. Weeks, Phys. Rev. B23, 2438 (1981).

[3] D.A. Huse and C.L. Henley, Phys. Rev. Lett. 54, 2708 (1985).

[4] M. Kardar, Phys. Rev. Lett. 55, 2235 (1985); M. Kardar and D.R. Nelson, Phys. Rev. Lett. 56, 472 (1986).

[5] R. Lipowski and M.E. Fisher, Phys. Rev. Lett. 56, 472 (1986).

[6] G. Forgacs, J.M. Luck, Th.M. Nieuwenhuizen and H. Orland, Phys. Rev. Lett. 572184 (1986).

[7] H. Li and M. Kardar, Phys. Rev. B42, 6546 (1990); M. Kardar and J.O. Indekeu, Europhys. Lett. 12, 61 (1990).

[8] P. Pfeifer, Y.J. Wu, M.W. Cole and J. Krim, sl Phys. Rev. Lett. 62, 1997 (1989); see also J. Krim, I. Heyvaert, C. Van Haesendonck and Y. Bruynseraede, Phys. Rev. Lett. 70, 57 (1993).

[9] G. Giugliarelli and A.L. Stella, Physica A 212, 12 (1994).

[10] B.B. Mandelbrot, Physica Scripta 32, 257 (1985).

[11] A. Crisanti, G. Paladin and A. Vulpiani, in "Products of Random Matrices in Statistical Physics", edited by H.K. Lotsch, Springer Series in Solid State Sciences, Vol.104 (Springer, Berlin, 1993).

[12] G. Forgacs, M.N. Svrakic and V. Privman, Phys. Rev. E37, 3818 (1988).

[13] Th.M. Nieuwenhuizen, J. Phys. A21, L567 (1988).

[14] G. Forgacs and Th.M. Nieuwenhuizen, J. Phys. A21, 3871 (1988).

[15] K. Hui and N. Berker, Phys. Rev. Lett. 62, 2507 (1989).

[16] J.O. Indekeu and J.M.J. van Leeuwen, Phys. Rev. Lett. 75, 1618 (1995).

[17] V. Privman, G. Forgacs and H.L. Frisch, Phys. Rev. B37, 9897 (1988).

[18] B. Derrida and R.B. Griffiths, Europhys. Lett. 8,111 (1989).

[19] I.M. Ward, in "Structure and Properties of Oriented Polymers", (Wiley, New York, 1975). 